\title{
Financial burden of heart failure in a developing country: cost analysis from Manipal Heart Failure Registry, India
}

\author{
Ajit Singh ${ }^{1} \cdot$ Sheetal Chauhan ${ }^{2} \cdot$ Tom Devasia $^{1} \cdot$ Yeshwanth Rao Karkala ${ }^{3} \cdot$ Ganesh Paramasivam $^{1} \cdot$ Prasad N. Shetty $^{1}$. \\ Deepak Uppunda ${ }^{1} \cdot$ Hashir Kareem ${ }^{4}$
}

Received: 14 June 2019 / Accepted: 6 September 2019 / Published online: 11 December 2019

(C) Springer-Verlag GmbH Germany, part of Springer Nature 2019

\begin{abstract}
Aim To estimate the comprehensive healthcare costs of heart failure (HF) and determine the utilization of healthcare resources (HRU) for 2 years following index hospitalization.

Subjects and methods The Manipal Heart Failure Registry (MHFR) is a prospective registry analyzing the financial burden and HRU in 610 patients with HF. Costs incurred by patients during 2 years following index hospitalization were estimated, and their association with socio-demographic and clinical factors were calculated. After 54 (8.8\%) in-hospital mortalities, 556 patients were followed up for 2 years.

Results The mean age of the study cohort was $65.08 \pm 13.6$ years, and $245(40.2 \%)$ were females. Based on the ejection fraction (EF), 506 (82.9\%) patients were diagnosed as having HF with reduced EF. Average hospital stay during index admission was 5.3 \pm 2.9 days. Total expenditure during index hospitalization was INR 36.3 million and during 2-year follow-up was INR 45.2 million. Average total expenditure per patient was INR 133,663. The average out-of-pocket expense was INR 82,766 and average health insurance coverage was INR 50,896. Difference in expenditure was significant $(P<0.05)$ between specific groups, i.e., etiologies, genders, HF phenotypes, age groups, and healthcare insurance types.

Conclusion Healthcare expenditure of patients with HF in India is much lower than for the western counterparts. Higher utilization of healthcare resources in HF patients with ischemic etiology, non-compliant to medications, and elderly (age $>60$ years) was associated with increased expenses. Interventional procedures and implantations account for the bulk of the expenses in ischemic HF patients.

Trial registration number Clinical Trial Registry of India: CTRI/2017/11/010395; National Institute of Health (NIH) clinical trial no.: NCT03157219.
\end{abstract}

Keywords Healthcare resource utilization $\cdot$ Expenditure $\cdot$ Economic burden $\cdot$ Costs $\cdot$ Developing country $\cdot$ Admissions

Electronic supplementary material The online version of this article (https://doi.org/10.1007/s10389-019-01141-w) contains supplementary material, which is available to authorized users.

Tom Devasia

tomdevasia@hotmail.com

Ajit Singh

ajitjsingh.mcops@gmail.com

Sheetal Chauhan

sheetal.ch9@gmail.com

Yeshwanth Rao Karkala

yashwanthrao2000@gmail.com

Ganesh Paramasivam

ganeshbmc@gmail.com

Prasad N. Shetty

dr.prasadnshetty@gmail.com
Deepak Uppunda

deepakuppunda18@gmail.com

Hashir Kareem

hashirkareem@gmail.com

1 Department of Cardiology, Kasturba Medical College, Manipal Academy of Higher Education, Manipal 576104, India

2 Department of Pharmacology, Melaka Manipal Medical College, Manipal Academy of Higher Education, Manipal 576104, India

3 Saint James School of Medicine, Lake City, Anguilla

4 Department of Cardiology, Kerala Institute of Medical Sciences, Trivandrum, Kerala, India 


\section{Background}

Heart failure (HF) is responsible for 1.8 million hospitalizations annually in India, and it affects $2-3 \%$ of the world's total population. HF is one of the leading medical causes of hospital admission among elderly population. Patients with HF admissions are very young ( 53 years) in India compared with $\sim 70$ years in the USA and Europe (Huffman and Prabhakaran 2010). The in-hospital HF mortality rate in India is 10-30.8\% compared with 4-7\% in Western countries (Seth et al. 2015; Callender et al. 2014). Heart failure is known to lead to frequent clinic visits because of worsening symptoms (Ambrosy et al. 2014).

Along with the adverse outcomes, HF is also known for its huge costs and financial burden for the patient, caregiver, society, and country budgets. HF represents $2-3 \%$ of the total cost of all medical conditions (Berry et al. 2001; Parissis et al. 2015). Globally, the estimated cost of HF in 2012 was $\$ 108$ billion accounting for $\sim \$ 65$ billion $(60 \%)$ of direct costs and $\sim$ $\$ 43$ billion (40\%) of indirect costs. India is the country in Southern Asia spending the most on HF according to the global data, estimating the amount as $\sim \$ 1186$ million (1.1\% of total global HF spending). This enormous cost of HF results from recurrent admissions, multiple drug therapy, device implantations, and various cardiac and non-cardiac comorbidities (Stewart et al. 2002). The economic burden of any disease constitutes two factors, direct and indirect costs. Direct costs of heart failure include physician/consultation charges, primary healthcare costs, and expenditure on hospitalization, hospital services, and medications followed by post-discharge expenses. Indirect costs include premature mortality, costs to society through morbidity, caregivers' related costs, and loss of productivity (Cook et al. 2014; Farmakis et al. 2015).

Heart failure is an important 'present day' problem with significant public health importance and financial implications for developing countries such as India. Both developed and developing countries spend a huge amount of their budgets for their citizens' healthcare. It is therefore important to do an elaborate cost estimation of HF management. An approximate estimation of the cost of HF management with optimal use of facilities will help health policy-makers to draw a broad outline regarding expenses per patient per year and the amount required to be kept aside for this group of patients. A comparison with developed countries would be revealing concerning how financial and health resource utilizations are being carried out in our center and also how developed countries could develop better cost-cutting plans with optimal patient results.

Only a few HF registry studies have been done in India, which followed the patients for less than a year, and therefore long-term data on disease management, healthcare resource utilization (HRU), and health economics have never been addressed adequately. Typically, these studies have focused primarily upon clinical profiles of hospitalized patients, where detailed long-term documentation of cardiac function, treatment patterns, outcomes, and healthcare expenditure is lacking. This study estimates the comprehensive healthcare costs of $\mathrm{HF}$ and determines the utilization of healthcare resources for 2 years. In this study we analyzed the total costs of management of different phenotypes of HF [heart failure with preserved ejection fraction (HFpEF) and heart failure with reduced ejection fraction (HFrEF)]. We designed the study in a manner to differentiate the costs in specific groups, which have not been well studied before, i.e., ischemic and nonischemic HF, males and females, HF with comorbidities and prevalent HF alone, and between different types of healthcare resource utilization groups (re-admissions vs. nonreadmissions; scheduled vs. unscheduled visits). This study is unique because to our knowledge it is the first and only study to date executed in this country for HF patients.

\section{Subjects and methods}

\section{Study design and setting}

The Manipal Heart Failure Registry (MHFR) is a prospective registry that documents the routine patterns of diagnosis and medical care for HF as well as treatment type and long-term clinical events including mortality and re-hospitalizations. The healthcare resource utilization and financial burden of disease were estimated as a secondary objective. Ethical approval was obtained from the Institutional Ethics Committee for MHFR.

The study recruited patients from a tertiary care center (the university's teaching hospital). Patients were directly presented or referred from all associated primary care hospitals/ clinics between January 2016 and December 2016. Patients who survived after index hospitalization were followed up for 2 years.

\section{Eligibility and sampling}

Patients with acute HF or acute exacerbation of chronic HF were included after the informed consenting process. The data of 610 patients were analyzed for financial burden domain between January 2016 and December 2016. After 54 (8.8\%) in-hospital mortalities, 556 patients were followed up for 2 years.

\section{Data collection}

\section{Socio-demographic and clinical information}

Socio-demographic details including gender, age, social habits, educational status, and income status of patients and 
caregivers were collected. Clinical characteristics, i.e., etiology, phenotypes of HF, New York Heart Association (NYHA) score at admission, and details of comorbid conditions were recorded for the analysis. Heart failure was divided into two categories based on the ejection fraction (EF), HFpEF with $\geq 50 \%$ and $\mathrm{HFrEF}$ with $<50 \%$. Drug utilization review was done for medications prescribed to the patients. Medication nonadherence was evaluated using a validated tool for all discharged patients during the follow-up period.

\section{Healthcare resource utilization}

We recorded the data that could influence the costs of HF starting from emergency department (ED) admission to discharge and at follow-ups. Interventions, device implantations, and cardiac diagnostic and therapeutic procedures including coronary angiogram $(\mathrm{CAG})$ and use of an intra-aortic balloon pump were noted in the recruited cohort. Utilization of a ventilator and intensive cardiac care unit and ward stays were also reported under healthcare resource utilization.

\section{Outcomes}

Post-discharge events, i.e., mortality, re-admissions, and scheduled and unscheduled visits, within the follow-up period were considered as outcomes.

\section{Index admission costs}

For this registry, we analyzed the total expense incurred during index hospitalization from in-patient bills. Overall index hospitalization expenditure was divided into five different costs: 'hospital services and consumables,' 'investigations,' 'medicines, procedural and devices,' 'consultation and professional charges,' and 'costs incurred by caregivers during admission.'

'Hospital services' included intensive care unit (ICU) costs, ward charges, admission procedure-related costs, and diets for patients. 'Consumables' comprised central sterile unit and general store materials. Investigations included biochemical, clinical/hematology, microbiology, radiology, and cardiac (electrocardiogram and echocardiogram) testing. 'Medicines, procedural and devices' comprised the costs of oxygen therapy and medicines including early treatment (injectables and infusions) of HF and drugs used during hospitalization procedures. It also consisted of interventions and device implantations. 'Professional charges' included the nursing costs, costs of an emergency visit by a clinician, and consultation costs of an HF specialist or other specialist needed to manage HF or HF-related comorbidities. 'Caregiver-related expenses' were calculated based on their current occupation and daily earnings. Travel charges and expenses for food and lodging were included as per the duration of caregiving during hospitalization.

\section{Follow-up costs}

Follow-up expenditure comprised the costs of re-admissions, medication expenses, scheduled and unscheduled visits to clinicians, and those related to caregivers. Re-admission costs again consisted of 'hospital services and consumables,' 'investigations,' 'medicines, procedural and devices,' 'consultation and professional charges,' and 'costs incurred by caregivers during admission.' Costs of scheduled and unscheduled visits included the consultation charges, hospital services (record maintenance and registration), investigations, and travel expenses. Caregiver's expenditure on follow-up involved travel charges, food and lodging, and loss of salary as per their occupation. Medication costs were estimated as per the bills presented by patients on follow-ups or the drug costs where patients were not able to produce the bills.

\section{Costs covered by health insurance}

Utilization of healthcare insurance which covered the HFrelated costs completely or partially was considered, and discounted costs were calculated. We divided the patients into two categories based on the insurance type and coverage: first, government healthcare schemes, private healthcare insurance, and uninsured patients; second, complete or partial reimbursement.

\section{Statistical analysis}

Data analysis was done using SPSS version 20 (SPSS Inc., IL, USA). Continuous data were presented as means and standard deviation. Proportions were reported for categorical data. Categorical variables were compared using the chi-square test and continuous variables through t-tests. Costs were compared using independent sample t-test between different groups, i.e., ischemic and non-ischemic heart failure, male and female patients, HFpEF and HFrEF, and scheduled and unscheduled visit groups. $P<0.05$ was considered statistically significant.

\section{Results}

\section{Socio-demographics and clinical characteristics}

A total of 610 patients were included in the study with mean age of $65.08 \pm 13.6$ years; $365(59.8 \%)$ were males. A caregiver was present for all the patients during index hospitalization and follow-up visits. Post-discharge care was given on a domiciliary basis to all the patients. No 
one was shifted to cardiac rehabilitation or any kind special care which increases the post-discharge costs. Of 610 patients, 196 (32.2\%) patients and 337 (55.2) caregivers were currently earning. Socio-demographic details are presented in Table 1.

At index hospitalization, NYHA functional class was assessed for the patients, and $354(58.1 \%)$ were class III. Based on EF, 506 (82.9\%) patients were diagnosed with HFrEF. Average hospital stay during index admission was $5.3 \pm 2.9$ days. Two hundred thirty-seven $(38.9 \%)$ patients presented with ischemia as the etiology of HF (as confirmed by electrocardiogram changes, troponin $\mathrm{T}$ values, and CAG report), followed by 149 (24.4\%) with cardiomyopathies of non-ischemic etiologies. Among comorbidities, hypertension was the major comorbidity in $316(51.8 \%)$ patients followed by $261(42.8 \%)$ and $257(42.1 \%)$ patients with anemia (hemoglobin $<12 \mathrm{~g} / \mathrm{dl}$ for males and $<11 \mathrm{~g} / \mathrm{dl}$ for females) and diabetes mellitus, respectively. Fifty-four (8.8\%) patients expired during index hospitalization, and 1- and 2-year composite mortalities were 146 (23.9\%) and 157 (25.7\%), respectively (Table 1).

\section{HF expenditure}

The total cost of HF management from index hospitalization for 610 patients was INR 81,534,469 (81.5 million) with an average 2-year cost of INR 133,663 per patient. After 8.8\% mortality, 556 patients were followed up for 2 years. Total expenditure for follow-up was computed as INR 45,244,349 (45.2 million) vs. INR 36,290,120 (36.3 million) for index hospitalization. The average cost paid by the patient was INR 82,766 after average health insurance coverage of INR 50,896.

In-hospitalization costs were less than half (44.6\%) of the total expenditure and comprised five categories as described in the methodology. Maximum costs of index admission were incurred through medications, procedures, and implantations and amounted to INR 23,753,461 (65.4\%), whereas consultation and professional charges amounted to only INR 1,316,502 (3.6\%) (Table 2). Two-year follow-up cost related to HF management was $55.4 \%$, and maximum expenditure incurred was from medicines $(38.3 \%)$ followed by readmissions (34.5\%) (Fig. 1).

\section{Comparison of costs between different groups}

Independent samples t-test was used to compare the average costs between specific groups such as phenotypes of HF, gender, etiology, medication compliance, and resources utilization. Patients with ischemic etiology, HFpEF, post-discharge re-hospitalizations due to HF, male gender, noncompliance to medications, and age $>60$ years were associated with additional expenses in HF costs with $p<0.05$ (Table 3 ).
Table 1 Socio-demographic and clinical characteristics of the study cohort

\begin{tabular}{|c|c|}
\hline Variables & Frequency $(\%)$ \\
\hline Age (years) & $65.08 \pm 13.6$ \\
\hline \multicolumn{2}{|l|}{ Age groups } \\
\hline 18-60 years & $210(34.4)$ \\
\hline$>60$ years & $400(65.6)$ \\
\hline Males & $365(59.8)$ \\
\hline Patients (earning) & $196(32.2)$ \\
\hline Caregivers (earning) & $337(55.2)$ \\
\hline \multicolumn{2}{|l|}{ Healthcare insurance } \\
\hline Government & $371(60.7)$ \\
\hline Private & $113(18.6)$ \\
\hline Uninsured & $126(20.7)$ \\
\hline Admission through ED & $329(54)$ \\
\hline Smoking & $198(32.5)$ \\
\hline Alcoholic & $189(31)$ \\
\hline \multicolumn{2}{|l|}{ Etiology } \\
\hline Ischemic heart disease & 237 (38.9) \\
\hline Cardiomyopathies & $149(24.4)$ \\
\hline VHD & $44(7.2)$ \\
\hline \multicolumn{2}{|c|}{ Procedures and interventions } \\
\hline CAG & $397(65.1)$ \\
\hline PTCA/CABG & $224(36.8)$ \\
\hline Devices & $16(2.6)$ \\
\hline IABP & $66(10.8)$ \\
\hline \multicolumn{2}{|l|}{ HF type } \\
\hline HFpEF & $104(17.1)$ \\
\hline HFrEF & $506(82.9)$ \\
\hline \multicolumn{2}{|l|}{ NYHA class } \\
\hline III & $354(58.1)$ \\
\hline IV & $149(24.4)$ \\
\hline \multicolumn{2}{|l|}{ Comorbid conditions } \\
\hline HTN & $316(51.8)$ \\
\hline Diabetes mellitus & $257(42.1)$ \\
\hline Anemia & $261(42.8)$ \\
\hline $\mathrm{CKD} / \mathrm{AKI}$ & $105(17.2)$ \\
\hline Arrhythmia (AF/VT) & $38(6.2)$ \\
\hline \multicolumn{2}{|l|}{ Index hospitalization } \\
\hline Duration of stay & $5.3 \pm 2.9$ days \\
\hline ICU stay & $512(84)$ \\
\hline Ward stay & $537(88)$ \\
\hline Ventilator support & $354(58)$ \\
\hline \multicolumn{2}{|l|}{ Outcomes } \\
\hline \multicolumn{2}{|l|}{ Mortality } \\
\hline In-hospital & $54(8.8)$ \\
\hline 2-year & $157(25.7)$ \\
\hline Re-admissions & $243(39.8)$ \\
\hline Visits to clinician & 2630 (4.7 per patient) \\
\hline Scheduled & 1058 (1.9 per patient) \\
\hline Unscheduled & 715 (1.3 per patient) \\
\hline Non-HF & 857 (1.4 per patient) \\
\hline
\end{tabular}


Table 1 (continued)

\begin{tabular}{ll}
\hline Variables & Frequency (\%) \\
\hline Drug utilization review & \\
Drugs per prescription & \\
In-hospital & 10.1 \\
Follow-up & 5.3 \\
Medication non-compliance & $106(17.3)$ \\
\hline
\end{tabular}

$\mathrm{ED}=$ Emergency department; HTN = hypertension; $\mathrm{VHD}=$ valvular heart disease; $\mathrm{CAG}=$ coronary angiogram; $\mathrm{PTCA}=$ percutaneous transluminal coronary angioplasty; $\mathrm{CABG}=$ coronary artery bypass grafting; $\mathrm{IABP}=$ intra-aortic balloon pump; $\mathrm{HFpEF}=$ heart failure with preserved ejection fraction; $\mathrm{HFrEF}=$ heart failure with reduced ejection fraction; $\mathrm{CKD}=$ chronic kidney disease; $\mathrm{AKI}=$ acute kidney injury; $\mathrm{AF}=$ atrial fibrillation; $\mathrm{VT}=$ ventricular tachycardia; $\mathrm{ICU}=$ intensive care unit

\section{Discussion}

HF is a complex clinical syndrome and a progressive disease with a high epidemiological burden. It causes repeated reoccurrences of symptoms, and it worsens with age resulting in frequent hospitalizations and high costs (Cho et al. 2018). Aging because of success against communicable disease in developing countries such as India increases the prevalence of HF. Urbanization and economic transition of a developing country increase average age ( $>60$ years), which escalates the HF burden (Huffman and Prabhakaran 2010). MHFR also supports the fact that the HF costs are high in the elderly (INR $110,734.0 \pm 33,478.2$ for $<60$ years vs. INR $145,700.8 \pm 56,765.2$ for $\geq 60$ years; $p=0.009$ ).

The HF costs are different for both genders; females show less expenditure than males. Males carry the additional burden of comorbidities such as diabetes and hypertension (Adhish et al. 2018). They are more prone to develop ischemiainduced $\mathrm{HF}$ at an earlier age than females. Females usually develop HF at a later age ( $>60$ years) because of menopause and late development of risk factors. The present study shows a significant difference between the HF costs for male (INR $158,384.2 \pm 34,262.2$ ) and female patients (INR 96,833.6 \pm 31,589.3); $p=0.002$.

Patients admitted with HFrEF are at increased risk of mortality and re-hospitalization. Among hospitalizations for heart failure, $\mathrm{HFpEF}$ represents a growing proportion and may overtake HFrEF as the predominant form of AHF (Steinberg et al. 2012). The cost difference between HF phenotypes was significant (INR 166,392.6 $\pm 35,487.2$ vs. INR $126,936.0 \pm 46,258.8 ; p=0.041)$. The index admission costs of HFrEF were more than those of HFpEF because of the ischemic etiology and interventions done, but long-term charges were much higher in HFpEF because of frequent non-ischemic hospitalizations and additional resource utilization (Murphy et al. 2017).
Per patient costs of ischemic HF was $180 \%$ more than for non-ischemic HF. A significant difference was noted in the costs for ischemic HF (INR 182,968.2 $\pm 33,562.0$ ) and nonischemic HF (INR 102,335.1 $\pm 25,689.2$ ); $p<0.001$. The main contributors to the high cost in ischemic HF were emergency room charges, procedural charges, implantation of drug-eluting stents (DES), and other interventions such as the use of an intra-aortic balloon pump (IABP) and ICU stay for post-procedural observations (Rihova et al. 2013). In the present study, CAG was done for 397 (65.1\%) patients with acute myocardial infarction or to rule out ischemic causes of heart failure. Among 237 (38.9\%) IHD patients, 224 (94.5) underwent percutaneous transluminal coronary angioplasty (PTCA) with DES or coronary artery bypass grafting (CABG), and 66 (27.8\%) patients needed IABP support.

Presence of comorbidities doubled the costs of HF. The costs for HF with comorbidities were significantly higher than for HF alone (INR 164,522.6 $\pm 43,234.2$ vs. INR 85,759.8 \pm $25,639.2 ; p<0.001)$. Coronary artery disease and hypertension are the major cardiac conditions, while renal insufficiency, anemia, and diabetes mellitus are well-known non-cardiac conditions in HF patients (Wexler et al. 2001). In the present study, half of the patients had hypertension, followed by diabetes mellitus, IHD, anemia, and renal diseases. Lio and his team did a regression model analysis for the costs, which showed $50 \%$ escalation in total HF costs because of renal diseases followed by $38 \%, 28 \%$, and $19 \%$ for coronary artery disease (CAD), diabetes, and hypertension, respectively (Liao et al. 2007). The presence of kidney disease has a strong influence on HF, leading to poor prognosis, i.e., mortality, re-admissions, and huge healthcare costs (House 2018). Chronic kidney disease (CKD) contributes to the development of HF risk factors including acid-base alterations, myocardial stunning, anemia, and uremic toxins (Tuegel and Bansal 2017). Management of renal insufficiency and associated risk factors increases the HF cost by 30-50\% (House 2018; Shiba and Shimokawa 2011). Diabetes mellitus is associated with re-admissions, poor outcomes, mortality, and increased costs of management (Cohen-Solal et al. 2008; Held et al. 2007; Bogner et al. 2010). Prevalence of anemia in HF patients is reported to be $30-55 \%$, which is more than what is reported in left ventricular dysfunction patients (Rocha et al. 2018). Increased resource utilization (testing and evaluation) and management of anemia increase the costs of HF (Allen et al. 2009).

The higher costs in re-admitted patients show more utilization of healthcare resources. Of the total 243 (39.8\%) patients readmitted, 406 times (1.7 per patients) was for HF management or the management of comorbid conditions. A significant difference was noted in the costs for patients who were readmitted (INR 179,452.6 $\pm 28,976.2$ ) and the patients who were not readmitted (INR 103,344.6 $\pm 29,768.2$ ) even once during the follow-up period; $p<0.001$. Patient readmission 
Table 2 Heart failure-related costs

\begin{tabular}{|c|c|c|c|}
\hline HF-related costs & Total cost in INR & $\begin{array}{l}\text { Average cost } \pm \text { SD } \\
\text { (INR) }\end{array}$ & $\begin{array}{l}\text { Average cost } \\
\text { (*USD) }\end{array}$ \\
\hline \multicolumn{4}{|l|}{ Index hospitalization $(N=610)$} \\
\hline Hospital services and consumables & $5,618,649.0$ & $9210.8 \pm 3408.2$ & $135.7 \pm 50.2$ \\
\hline Costs of ICU stay & $1,221,037.0$ & & \\
\hline Ward charges & $854,488.0$ & & \\
\hline Cardiac monitoring & $1,652,551.0$ & & \\
\hline CSU/general store materials & $1,458,083.0$ & & \\
\hline Other services & $432,490.0$ & & \\
\hline Investigations & $3,943,650.0$ & $6465.0 \pm 2320.3$ & $85.3 \pm 34.2$ \\
\hline Biochemistry & $2,439,390.0$ & & \\
\hline Clinical/hematological & $399,733.0$ & & \\
\hline Radiology & $188,856.0$ & & \\
\hline Cardiology (ECG/ECHO) & $915,671.0$ & & \\
\hline Medications, procedures, and devices & $23,753,461.0$ & $38,940.1 \pm 21,650.2$ & $573.9 \pm 319.1$ \\
\hline Medications & $6,179,117.0$ & & \\
\hline Procedural charges & $9,117,060.0$ & & \\
\hline Devices/implantations/DES & $8,457,284.0$ & & \\
\hline Consultation and professional charges & $1,316,502.0$ & $2158.2 \pm 768.3$ & $31.8 \pm 11.3$ \\
\hline Consultation charges & $545,218.0$ & & \\
\hline Costs of nursing & $771,284.0$ & & \\
\hline Caregiver-related costs & $1,657,858.0$ & $2717.8 \pm 1098.2$ & $40.1 \pm 16.2$ \\
\hline Total cost of index admission & $36,290,120.0$ & $59,492.0 \pm 25,465.8$ & $876.8 \pm 375.3$ \\
\hline Costs covered by health insurance & $17,019,793.0$ & & \\
\hline Total out-of-pocket costs & $19,270,327.0$ & & \\
\hline \multicolumn{4}{|l|}{ Follow-up $(N=556)$} \\
\hline Visit to clinicians & $9,380,515.0$ & $16,871.4 \pm 7860.2$ & $248.7 \pm 115.8$ \\
\hline HF-scheduled & $3,930,470.0$ & & \\
\hline HF-unscheduled & $2,656,225.0$ & & \\
\hline Non-HF visits & $2,793,820.0$ & & \\
\hline Re-admissions & $15,608,314.0$ & $28,072.5 \pm 14,560.1$ & $413.7 \pm 214.6$ \\
\hline Cardio HF-related & $4,947,144.0$ & & \\
\hline Cardio non-HF & $8,079,640.0$ & & \\
\hline Non-cardio non-HF & $2,581,530.0$ & & \\
\hline Costs of medicines & $17,314,440.0$ & $31,141.1 \pm 9867.2$ & $459.0 \pm 145.4$ \\
\hline Caregiver-related costs & $2,941,080.0$ & $5289.7 \pm 1364.2$ & $77.9 \pm 20.2$ \\
\hline Total follow-up cost & $45,244,349.0$ & $81,374.7 \pm 22,349.2$ & $1199.3 \pm 329.4$ \\
\hline Costs covered by heath insurance & $14,026,847.0$ & $25,228.1 \pm 9878.0$ & $371.8 \pm 145.6$ \\
\hline Out-of-pocket costs & $31,217,502.0$ & $56,146.6 \pm 17,698.2$ & $827.5 \pm 260.8$ \\
\hline Total HF expenditure & $81,534,469.0$ & $133,663.1 \pm 36,554.2$ & $1970.1 \pm 538.7$ \\
\hline Total costs covered by insurance & $31,046,640.0$ & $50,896.1 \pm 14,682.3$ & $750.1 \pm 216.4$ \\
\hline Out-of-pocket costs & $50,487,829.0$ & $82,766.9 \pm 26,583.0$ & $1219.9 \pm 391.8$ \\
\hline
\end{tabular}

Bold emphasis in table 2 represents the total costs or the sum of costs given for subgroups

$\mathrm{INR}=$ Indian rupee; $\mathrm{CSU}=$ central sterile unit $\mathrm{ECG}=$ electrocardiogram; $\mathrm{ECHO}=$ echocardiogram; $\mathrm{ICU}=$ intensive care unit; DES = drug-eluting stents

*INR was converted into USD rates at the time of analysis $(1 \mathrm{USD}=67.85 \mathrm{INR})$ was associated with the severity of the condition and acute worsening of HF symptoms where a patient needed urgent hospitalization. Emergency treatment increases the related costs by the use of circulatory support, intensive and cardiac services, invasive diagnostic and therapeutic procedural charges, and the total length of stay (Philbin et al. 2001). A difference was noted even between the expenditures for patients who needed ventilator support during index admission 


\section{HF Expenditure}

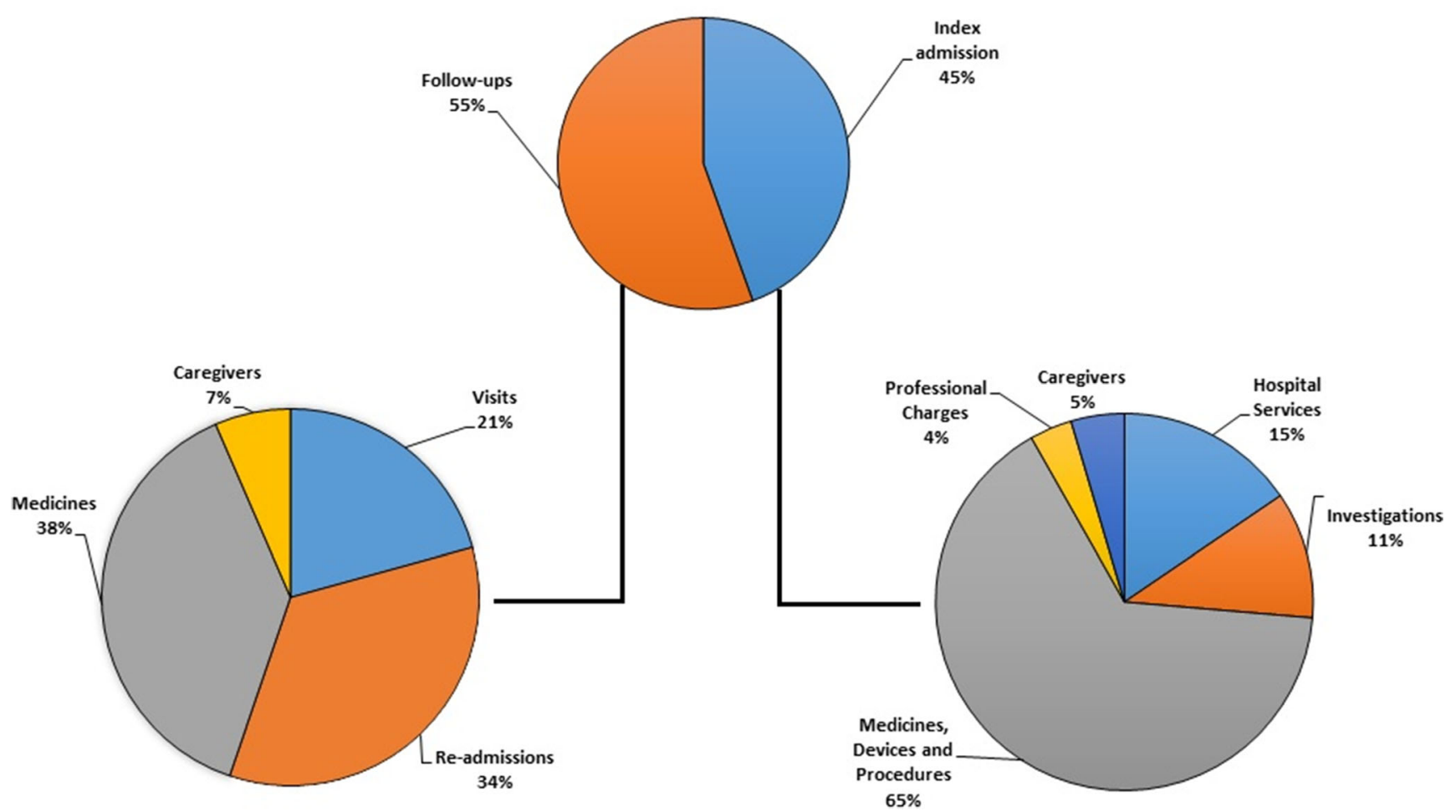

Fig. 1 Heart failure cost distribution. Pie charts show the overall heart failure costs and their distribution into costs during index admission and a 2-year follow-up period

and those who did not (INR 143,356.6 $\pm 38,744.3$ vs. INR $120,246.3 \pm 37,829.2 ; p=0.039)$. Repeated non-cardiac consultations for comorbidity management increased the costs too.

Medication non-adherence is positively related to major adverse cardiac events (MACEs) including cardiac death and recurrent ischemic events, which worsen the prognosis of HF and increase expenditures. After hospital discharge, 556 patients were assessed for medication non-compliance, and $106(19.1 \%)$ were found non-compliant for medications. Costs were compared for all 610 patients, and statistical significance was noted between compliant (INR 129,034.1 \pm $43,988.1$ ) and non-compliant (INR 155,672.5 $\pm 28,772.4$ ) patients; $p=0.013$. Drug non-compliance is a serious issue especially in developing countries such as India where patients try to reduce expenditures by stopping medicines without consulting physicians. However, it is important to know that acute decompensation following drug non-compliance will actually lead to life-threatening scenarios such as acute pulmonary edema and increased financial burden. It is recommended that for financially underprivileged patients, government and/or the sponsoring authorities consider supplying daily medicines free of cost or at subsidized rates to avoid the overall longterm financial burden.

Risk factors for cardiovascular diseases such as smoking, excess alcohol consumption, obesity, and physical inactivity are equally responsible for HF worsening, and it is again associated with high healthcare resource utilization, re-admissions, and increased expenditure (Cook et al. 2014). Timely management of comorbidities and risk factors of HF such as anemia, renal insufficiency, and valvular heart disease (VHD) can reduce the mortality rate, re-admissions, and financial burden.

Proper utilization of healthcare resources such as ED, ICU, ventilator support, and on-time HF expert visit and interventions could be a supportive measure to reduce the total costs. First evaluation of HF for all patients was done at the ED or out-patient department (OPD), and then the patients were shifted to the ICU or wards. Three hundred twenty-nine (54\%) patients primarily presented to the ED and had a noticeable difference in the costs compared with the patients who presented to the out-patient department (INR 146,931.1 \pm $42,999.2$ vs. INR $118,024.6 \pm 35,923.2 ; p=0.022$ ). In MHFR, average ED stay for the study cohort was $2 \mathrm{~h}$ $40 \mathrm{~min}$, which was statistically associated with in-hospital mortality. Patients with decompensated HF and worsened conditions were admitted through the ED, and they used maximum healthcare resources including ventilator and ICU stay and initial therapy with injectable diuretics and inotrope infusions. Poor prognosis in the patients admitted through the ED increased the costs. It was observed that controlling fluid and salt intake and monitoring weight were associated with 
Table 3 Comparison of total heart failure costs between different groups

\begin{tabular}{|c|c|c|c|c|c|}
\hline Variables & Frequency $(\%)$ & Total costs & $\begin{array}{l}\text { Cost per patient in } \\
\text { INR }\end{array}$ & $\begin{array}{l}\text { Costs per patient } \\
\text { USD }^{*}\end{array}$ & $P$ value \\
\hline \multicolumn{6}{|l|}{ Gender } \\
\hline Male & $365(69.8)$ & $57,810,232.0$ & $158,384.2 \pm 34,262.2$ & $2334.3 \pm 505.1$ & \multirow[t]{2}{*}{0.002} \\
\hline Female & $245(40.2)$ & $23,724,237.0$ & $96,833.6 \pm 31,589.3$ & $1427.2 \pm 465.6$ & \\
\hline \multicolumn{6}{|l|}{ Age groups (years) } \\
\hline$<60$ & $210(34.4)$ & $23,254,140.0$ & $110,734.0 \pm 33,478.2$ & $1632.0 \pm 493.4$ & \multirow[t]{2}{*}{0.009} \\
\hline$\geq 60$ & $400(65.6)$ & $58,280,329.0$ & $145,700.8 \pm 56,765.2$ & $2147.4 \pm 836.6$ & \\
\hline \multicolumn{6}{|c|}{ Heart failure categories } \\
\hline HFpEF & $104(17.1)$ & $17,304,830.4$ & $166,392.6 \pm 35,487.2$ & $2452.4 \pm 523.0$ & \multirow[t]{2}{*}{0.041} \\
\hline HFrEF & $506(82.9)$ & $64,229,638.6$ & $126,936.0 \pm 46,258.8$ & $1870.8 \pm 681.8$ & \\
\hline \multicolumn{6}{|c|}{ Ischemic vs. non-ischemic } \\
\hline Ischemic HF & $237(38.8)$ & $43,363,463.4$ & $182,968.2 \pm 33,562.0$ & $2696.7 \pm 494.6$ & \multirow[t]{2}{*}{$<0.001$} \\
\hline Non-ischemic HF & $373(61.2)$ & $38,171,005.6$ & $102,335.1 \pm 25,689.2$ & $1508.3 \pm 378.6$ & \\
\hline \multicolumn{6}{|c|}{ Medication noncompliance } \\
\hline Compliant & $504(82.6)$ & $65,033,184.0$ & $129,034.1 \pm 43,988.1$ & $1909.8 \pm 648.3$ & \multirow[t]{2}{*}{0.013} \\
\hline Non-compliant & $106(17.4)$ & $16,501,285.0$ & $155,672.5 \pm 28,772.4$ & $2294.4 \pm 424.1$ & \\
\hline \multicolumn{6}{|c|}{ Readmissions within 2 years (any) } \\
\hline Readmission & $243(39.8)$ & $43,606,988.3$ & $179,452.6 \pm 28,976.2$ & $2644.8 \pm 427.1$ & \multirow[t]{2}{*}{$<0.001$} \\
\hline No readmissions & $367(60.2)$ & $37,927,480.7$ & $103,344.6 \pm 29,768.2$ & $1523.1 \pm 438.7$ & \\
\hline \multicolumn{6}{|c|}{ Ventilator support during index admission } \\
\hline Yes & $354(58.0)$ & $50,751,422.4$ & $143,356.6 \pm 38,744.3$ & $2112.8 \pm 571.0$ & \multirow[t]{2}{*}{0.039} \\
\hline No & $256(42.0)$ & $30,783,046.6$ & $120,246.3 \pm 37,829.2$ & $1772.2 \pm 557.5$ & \\
\hline \multicolumn{6}{|l|}{ First evaluation } \\
\hline $\mathrm{ED}$ & $330(54.1)$ & $48,487,593.0$ & $146,931.1 \pm 42,999.2$ & $2165.5 \pm 633.7$ & \multirow[t]{2}{*}{0.022} \\
\hline OPD & $280(45.9)$ & $33,046,876.0$ & $118,024.6 \pm 35,923.2$ & $1739.5 \pm 529.5$ & \\
\hline \multicolumn{6}{|l|}{ Comorbidities } \\
\hline Yes & $371(60.8)$ & $61,037,876.8$ & $164,522.6 \pm 43,234.2$ & $2424.8 \pm 637.2$ & \multirow[t]{2}{*}{$<0.001$} \\
\hline No & $239(39.2)$ & $20,496,592.2$ & $85,759.8 \pm 25,639.2$ & $1264.0 \pm 377.9$ & \\
\hline \multicolumn{6}{|l|}{ Insurance types } \\
\hline Government & $371(60.7)$ & $50,115,701.8$ & $135,082.7 \pm 43,769.0$ & $1990.9 \pm 645.1$ & \multirow[t]{3}{*}{0.002} \\
\hline Non-government & $113(18.6)$ & $18,839,721.6$ & $166,723.2 \pm 38,992.2$ & $2457.2 \pm 574.7$ & \\
\hline Uninsured & $126(20.7)$ & $12,579,046.2$ & $99,833.7 \pm 23,654.2$ & $1471.4 \pm 348.6$ & \\
\hline
\end{tabular}

$\mathrm{INR}=$ Indian rupee; $\mathrm{HFpEF}=$ heart failure with preserved ejection fraction; $\mathrm{HFrEF}=$ heart failure with reduced ejection fraction; $\mathrm{ED}=$ emergency department; $\mathrm{OPD}=$ out-patient department

*INR was converted into USD rates at the time of analysis ( $1 \mathrm{USD}=67.85 \mathrm{INR})$

$\mathrm{P}$ value represents the significance of cost difference between groups positive outcomes (decreased mortality and re-admission rates). Education and proper counseling sessions for patients and caregivers to manage $\mathrm{HF}$ at home decrease the frequency of unscheduled visits and re-admissions, which are major components of the total costs.

Optimal use of guideline-directed medical therapy (GDMT) and evidence-based and community-based preventive programs can reduce costly HF hospitalizations (Liao et al. 2007). HF has a significant impact on the national economy and social security system. As we previously mentioned, HF covers $2 \%$ of the total healthcare expenditures, and more than two-thirds of it go to hospitalizations. Preventable factors such as HF-related hospitalizations, training to treat risk factors, and adherence to guidelines and medications can reduce the additional burden.

Various types of health insurance are offered to Indians by the Indian government and private firms. The payment system differs as some insurances pay the costs during hospitalization and others reimburse the expenses later. Some completely cover the expenses, and others give partial reimbursement. Types of insurance schemes impact costs differently (Ding et al. 2017). In our study cohort, there was a non-negligible difference in HF costs in government insured, nongovernment insured, and uninsured patients (INR 135,082.7 
$\pm 43,769.0$ vs. INR $166,723.2 \pm 38,992.2$ vs. INR 99,833.7 \pm 23,654.2; $p=0.002$ ). Proper selection of medical insurance and monitoring by governing bodies can reduce medical costs. Superfluous utilization of healthcare resources in patients with medical insurance also hikes the HF costs. This can be reduced after appropriate management by the clinician and other stakeholders.

Overall HF-related costs are much less in India than in the European countries and US. Costs from index hospitalization to follow-up are 5-10 times more in the western counterparts (Ambrosy et al. 2014).

\section{Study limitations}

The study was conducted in a teaching hospital of a university located in a semi-urban area. The costs of hospital services, procedural charges, and professional charges were less than in the urban set-ups. We could not directly compare the HF burden at our center with hospitals in urban areas. The study did not analyze all the indirect disease costs, i.e., productivity loss, costs of morbidity for society, caregiver's distress, and familyrelated financial concerns because of the HF patient's condition.

\section{Conclusions}

The study concluded that ischemic etiology, male gender, and age $>60$ years are associated with additional HF costs. Male gender is linked with an extra burden of risk factors and comorbidities which increase the total expenditure. Interventional procedures and implantations account for the bulk of the expenses in ischemic HF patients. Aging increases the HF burden along with frequent hospitalizations resulting in increased costs. High healthcare resource utilization during re-admissions and scheduled and unscheduled visits is associated with increased HF costs. HFpEF group, admission through the ED, and utilization of a ventilator during hospitalizations are also significantly associated with high costs. Drug non-compliance is an important and easily avoidable cause of increased healthcare expenditure. Comorbidities, i.e., hypertension, diabetes mellitus, anemia, and renal insufficiencies, are highly associated with increased HF costs. Type of medical insurance has a different impact on resource utilization and total expenditures. Proper selection and monitoring of insurance schemes will help reduce the costs. Healthcare expenditure of patients with HF in India is much lower than in the western counterparts.

Acknowledgements Manipal Heart Failure Registry (MHFR) is supported by the Manipal Academy of Higher Education (MAHE) by means of facilities needed to conduct the study. No external/industry funds were received for the current study.

\section{Compliance with ethical standards}

Ethical considerations The study was approved by the Institutional Ethics Committee of Manipal Academy of Higher Education (MAHE) and the identification number was MUEC/20/2015-16. An informed consent form was duly signed by participants and the investigator before commencement of the study, and all procedures were carried out following the 1964 Helsinki Declaration.

Conflict of interest The authors declare that they have no conflict of interest.

\section{References}

Adhish S, Husaini B, Taira D, Norris K, Moonis M, Levine R (2018) Depression effects on hospital cost of heart failure patients in California: an analysis by ethnicity and gender. Indian $\mathrm{J}$ Community Med 43(1):49

Allen LA, Anstrom KJ, Horton JR, Shaw LK, Eisenstein EL, Felker GM (2009) Relationship between anemia and health care costs in heart failure. J Card Fail 15(10):843-849

Ambrosy AP, Fonarow GC, Butler J et al (2014) The global health and economic burden of hospitalizations for heart failure: lessons learned from hospitalized heart failure registries. J Am Coll Cardiol 63:1123-1133

Berry C, Murdoch DR, McMurray JJ (2001) Economics of chronic heart failure. Eur J Heart Fail 3:283-291

Bogner H, Miller S, de Vries H, Chhatre S, Jayadevappa R (2010) Assessment of cost and health resource utilization for elderly patients with heart failure and diabetes mellitus. J Card Fail 16(6): 454-460

Callender T, Woodward M, Roth G et al (2014) Heart failure care in lowand middle-income countries: a systematic review and meta-analysis. PLoS Med 11(8):e1001699

Cho H, Oh S, Lee H, Cho H, Kang H (2018) The incremental economic burden of heart failure: a population-based investigation from South Korea. PLoS One 13(12):e0208731

Cohen-Solal A, Beauvais F, Logeart D (2008) Heart failure and diabetes mellitus: epidemiology and management of an alarming association. J Card Fail 14(7):615-625

Cook C, Cole G, Asaria P, Jabbour R, Francis D (2014) The annual global economic burden of heart failure. Int J Cardiol 171(3):368-376

Ding J, Zhang X, Hu X, Chen H, Yu M (2017) Analysis of hospitalization expenditures and influencing factors for inpatients with coronary heart disease in a tier-3 hospital in Xi'an, China. Medicine 96(51):e9341

Farmakis D, Filippatos G, Parissis J, Lekakis J (2015) The social and economic burden of hospitalization for heart failure. Medicographia 37(2):135-138

Held C, Gerstein H, Yusuf S et al (2007) Glucose levels predict hospitalization for congestive heart failure in patients at high cardiovascular risk. Circulation 115(11):1371-1375

House A (2018) Management of Heart Failure in advancing CKD: Core curriculum 2018. Am J Kidney Dis 72(2):284-295

Huffman MD, Prabhakaran D (2010) Heart failure: epidemiology and prevention in India. Natl Med J India 23(5):283-288

Liao L, Anstrom K, Gottdiener J et al (2007) Long-term costs and resource use in elderly participants with congestive heart failure in the cardiovascular health study. Am Heart J 153(2):245-252

Murphy T, Waterhouse D, James S et al (2017) A comparison of HFrEF vs HFpEF's clinical workload and cost in the first year following hospitalization and enrollment in a disease management program. Int J Cardiol 232:330-335 
Parissis J, Athanasakis K, Farmakis D et al (2015) Determinants of the direct cost of heart failure hospitalization in a public tertiary hospital. Int J Cardiol 180:46-49

Philbin EF, Mccullough PA, Dec GW, Disalvo TG (2001) Length of stay and procedure utilization are the major determinants of hospital charges for heart failure. Clin Cardiol 24(1):56-62

Rihova B, Parenica J, Jarkovsky J et al (2013) Cardiology department hospitalization costs in patients with acute heart failure vary according to the etiology of the acute heart failure: data from the AHEAD Core registry 2005-2009. Cor et Vasa 55(1):e7-e14

Rocha BM, Cunha GJ, Falcão LF (2018) The burden of iron deficiency in heart failure: therapeutic approach. J Am Coll Cardiol 71(7):782-793

Seth S, Khanal S, Ramakrishnan S, Gupta N, Bahl VK (2015) Epidemiology of acute decompensated heart failure in India: the AFAR study (acute failure registry study). J Pract Cardiovasc Sci $1: 35-38$

Shiba N, Shimokawa H (2011) Chronic kidney disease and heart failure-bidirectional close link and common therapeutic goal. J Cardiol 57(1):8-17

Steinberg B, Zhao X, Heidenreich P et al (2012) Trends in patients hospitalized with heart failure and preserved left ventricular ejection fraction. Circulation 126(1):65-75

Stewart S, Jenkins A, Buchan S, McGuire A, Capewell S, McMurray JJ (2002) The current cost of heart failure to the National Health Service in the UK. Eur J Heart Fail 4:361-371
Tuegel C, Bansal N (2017) Heart failure in patients with kidney disease. Heart 103(23):1848-1853

Wexler D, Chen J, Smith G et al (2001) Predictors of costs of caring for elderly patients discharged with heart failure. Am Heart J 142(2): $350-357$

\section{Key points for decision-makers}

1. HF-related healthcare expenditure in India is much lower than in the western counterparts. Interventional procedures and implantations account for the bulk of the expenses. Optimum utilization of healthcare resources and adherence to therapy guidelines can help in controlling the total costs of HF.

2. Adherence to the drug therapy may help in reducing the rate of readmissions and mortality resulting in a reduction of HF-related expenses. Timely management of cardiovascular risk factors may reduce the economic burden of HF.

Publisher's note Springer Nature remains neutral with regard to jurisdictional claims in published maps and institutional affiliations. 